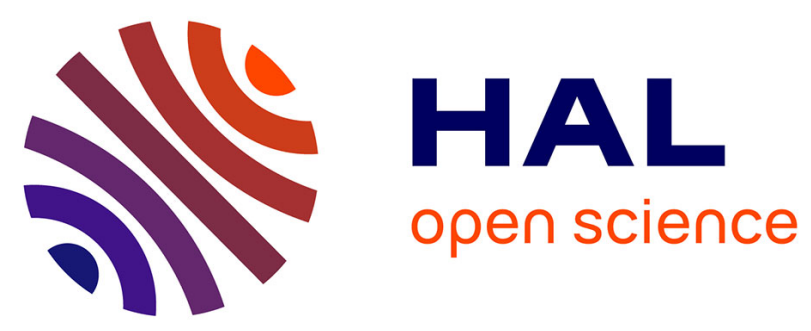

\title{
Classification of classical non-Gaussian noises with respect to their detrimental effects on the evolution of entanglement using a system of three-qubit as probe
}

Lionel Tenemeza Kenfack, Tchoffo Martin, Lukong Cornelius Fai

\section{- To cite this version:}

Lionel Tenemeza Kenfack, Tchoffo Martin, Lukong Cornelius Fai. Classification of classical nonGaussian noises with respect to their detrimental effects on the evolution of entanglement using a system of three-qubit as probe. 2017. hal-01612776

\section{HAL Id: hal-01612776 \\ https://hal.science/hal-01612776}

Preprint submitted on 8 Oct 2017

HAL is a multi-disciplinary open access archive for the deposit and dissemination of scientific research documents, whether they are published or not. The documents may come from teaching and research institutions in France or abroad, or from public or private research centers.
L'archive ouverte pluridisciplinaire HAL, est destinée au dépôt et à la diffusion de documents scientifiques de niveau recherche, publiés ou non, émanant des établissements d'enseignement et de recherche français ou étrangers, des laboratoires publics ou privés. 


\title{
Classification of classical non-Gaussian noises with respect to their detrimental effects on the evolution of entanglement using a system of three-qubit as probe
}

Laboratoire De Matière Condensée, D'électronique Et De Traitement Du Signal (LAMACETS), Département de Physique, Faculté des Sciences, Université de Dschang, BP 67 Dschang, Cameroun.

\begin{abstract}
We use a system of three non-interacting qubits as a quantum probe to classify three classical non-Gaussian noises (namely the static, colored (pink and brown) and random telegraph noise), according to their detrimental effects on the evolution of entanglement of the latter. The probe system is initially prepared in the GHZ state and coupled to the noises in independent environments. Seven configurations for the qubit-noise coupling (QNC) are considered. To estimate the destructive influence of each kind of noise, we employ the tripartite negativity to compare the evolution of entanglement in these QNC configurations to each other with the same noise parameters. The results show that the evolution of entanglement is drastically impacted by the QNC configuration considered as well as the properties of the environmental noises and that the SN is more detrimental to the survival of entanglement than the RTN and CN, regardless of the Markov or non-Markov character of the RTN and the color of the CN. We also observed that pink noise is more fatal to the system than the RTN and that the situation is totally reversed in the case of brown noise. Finally, we show that these noises, in descending order of destructive influence, can be classified as follows: $\mathrm{SN}>$ pink noise $>$ RTN $>$ brown noise.
\end{abstract}

Keywords: Classical noise, Non-Gaussian noise, Entanglement, Qubit.

\section{Introduction}

Quantum entanglement is one of the most remarkable properties of quantum systems. It has been defined in 1935 by Erwin Schrdinger [1] as one of the most peculiar phenomenon of quantum mechanics in which the global states of a compound quantum system cannot be written as product of the states of individual subsystems [2]. It has been demonstrates that quantum entanglement is a fundamental an indispensable resource for many potential applications in the field of quantum information theory such as for instance the quantum cryptography [3, 4] and information processing [5 7]. Unfortunately, real quantum system cannot refrain from interacting with its external environment (the so-called decoherence) which introduces noise in the system and which, in turn, results into the destruction of the quantum properties of the system and consequently make its unusable. However, it has been recognized that decoherence may play a nondetrimental role on the evolution of entanglement existing among different marginal parts of a quantum system [8, 9].

The interaction of a quantum system with its external environment may be simulated either classically or quantum mechanically. In the former, the system and the environment is look as a single quantum entity governed by an overall unitary operator; it is worth nothing that this description is more realistic because it includes the transfer of information and it is also related to the idea that the phenomenon of decoherence is

*kenfacklionel300@gmail.com 
strongly connected to the entanglement between the quantum system and its external environment. On the other hand, in the classical approach (also known as random classical force (RCF) approach), the system and the environment is considered as a quantum system coupled to random classical forces. Contrary to the first description, in the RCF approach, the system is not entangled with the environment. Furthermore, it is important to note that when the environment is very complex (with many degree of freedom); the quantum approach becomes very challenging. In such a situation the RCF approach represents a valid and reliable alternative to overcome the challenge. Nevertheless, for certain system-environment (SE) interactions, it has been pointed out that it is possible to obtain a classical simulation that is completely equivalent to the quantum simulation [10 15$]$.

Motivated by the desire to minimize the detrimental effects of decoherence on the properties of quantum systems (e.g., quantum entanglement), the effects of different classical environments characterized either by Gaussian or non-Gaussian distributed noise on the evolution of many quantum systems (e.g., small dimensional systems or qubits) have been intensely investigated in recent years both theoretically [16 30] and experimentally [31, 32]. So far, interesting results have been obtained. For instance, it has been proved in 20-26] that the indirect interaction between the subsystems of a composite system due to their coupling to a common classical environmental noise (CEN) can enable to preserves their entanglement indefinitely. Besides, the effects of the input configuration of the system as well as the QNC configuration on the evolution of entanglement have been investigated both for two- and three-qubit systems [16 26]. On the other hand, the joint effects of several kinds of CENs on the evolution of entanglement have also been investigated both for two- and three-qubit system [22, 23]. While most of these studies are devoted to the possibility of protecting entanglement via the QNC configuration and the initial input state, little attention has been paid to classify different kinds of CENs with respect to their detrimental effects on the evolution of entanglement. For example is has been shown that when the subsystem of a composite system are coupled to a CEN in a common environment, entanglement is better preserves than when there are coupled either in mixed or independent environments [16-26]. In other words, even if the effects of many kinds of CENs on the evolution of entanglement have been widely investigated in the literature, the classification of these CENs with respect to their harmful impact on the evolution of entanglement has not yet been investigated.

In this paper, we intend to use a three-qubit system coupled in independent environments and subjected to different CENs, as a quantum probe to classify these CENs with respect to their detrimental effects on the evolution of entanglement of the probe. In particular, three different kinds of CENs namely the RTN, SN and CN are considered. In the case of RTN, two particular regimes namely the fast or Markov RTN and slow or non-Markov RTN are considered while for CN, we consider both the case of pink and brown noise. Our approach to classify these CENs involves seven QNC configurations: in the first QNC configuration, we consider that the first qubit of the probe system is coupled with the RTN, the second with the SN and the third with the $\mathrm{CN}$ and this is denoted (RTN;SN;CN); the other QNC configurations are obtained from the latter by switching each qubits local noise by the local noises of the remaining two qubits. Concretely speaking, the second and third QNC configuration denoted (SN;SN;CN) and (CN;SN;CN) are obtained from the first one by considering that the first qubit is no longer coupled with the RTN, but rather with either the SN or RTN. In the same way, the fourth and fifth QNC configuration denoted (RTN;RTN;CN) and $(\mathrm{RTN} ; \mathrm{CN} ; \mathrm{CN})$ are obtained from the first QNC configuration by switching the SN to which the second qubit of the probe is coupled either by the RTN or the CN. Finally, the sixth and seventh QNC configuration denoted (RTN;SN;RTN) and (RTN;SN;SN) are derived from the first one by switching the CN to which the third qubit of the probe system is coupled either by the RTN or the SN. It is worth recalling that the notation $(\mathrm{XX} ; \mathrm{YY} ; \mathrm{ZZ})$ means that the first qubit of the probe system is locally coupled to XX, the second to $\mathrm{YY}$ and the third to ZZ. By employing the tripartite negativity as entanglement measure, we compare the dynamics of entanglement in the seven QNC configurations to each other. These comparisons allow us to appreciate the destructive influence of the presence or absence of each kinds of CEN on the evolution of entanglement which thereafter should enable us to classify these noises with respect to their detrimental effects. The probe is initially prepared in the GHZ state and each CEN is introduced by means of a stochastic process. Therefore, the time evolution of the probe system is obtained by performing an ensemble average over all the possible realizations of each stochastic process and the entanglement is quantified by means of tripartite negativity. 
The rest of the paper is organized as follows: In Sec. 2, we present the physical model and introduce the tripartite negativity. The analytical results as well as the behaviors of entanglement for the different QNC configurations are presented in Sec. 3, Finally, we summarize our result in Sec. 4.

\section{The physical model}

Our model, similar to those considered in 2026 , consists of three noninteracting qubits coupled in independent environments in which each qubit interacts with a specific CEN. As we have already pointed out, three different kinds of CEN namely RTN, SN and CN are considered. In order to fully classify these CENs with respect to their detrimental effects on the evolution of entanglement of the probe system (threequbit system), seven QNC configurations namely respectively (RTN;SN;CN), (SN;SN;CN), (CN;SN;CN), (RTN;RTN;CN), (RTN;CN;CN), (RTN;SN;RTN) and (RTN;SN;SN) are considered. In these configurations, the dynamics of the system is governed by the following Hamiltonian

$$
\mathcal{H}(t)=\mathcal{H}_{1}(t) \otimes \mathbb{I}_{2} \otimes \mathbb{I}_{3}+\mathbb{I}_{1} \otimes \mathcal{H}_{2}(t) \otimes \mathbb{I}_{3}+\mathbb{I}_{1} \otimes \mathbb{I}_{2} \otimes \mathcal{H}_{3}(t),
$$

where, $\mathbb{I}_{k}, k=1,2,3$ denotes the identity operator in the subspace of the qubits $k$; and $\mathcal{H}_{k}(t)$ stands for the single-qubit Hamiltonian which contains a stochastic variable giving rise to the external noise and can be explicitly expressed as

$$
\mathcal{H}_{k}(t)=\epsilon_{k, 0} \mathbb{I}_{k}+g_{k} \vartheta_{k}^{s}(t) \sigma_{k}^{x},
$$

where $\epsilon_{k, 0}$ is the degenerated qubits energy in the absence of noise, $\mathbb{I}_{k}$ and $\sigma_{k}^{x}$ are respectively the identity and the spin-flip Pauli matrix acting on the subspace of the qubit $k . g_{k}$ characterizes the strength of interaction between qubit $k$ and its local CEN. $\vartheta_{k}^{s}(t)(s \in\{R T N, S N, C N\})$ stands for a stochastic parameter whose statistic depends upon the kind of CEN to which the qubit $k$ is coupled. More precisely, when the qubit is coupled to the SN, the stochastic parameter $\vartheta_{k}^{s}(t) \equiv \vartheta_{k}^{S N}(t)$ is assumed to be independent on time and characterized by the following flat probability distribution [33, 34]

$$
\mathrm{P}\left(\vartheta_{k}^{S N}\right)=\left\{\begin{array}{l}
\frac{1}{\eta_{m}} \longrightarrow\left|\vartheta_{k}^{S N}(t)-\eta_{0}\right| \leq \frac{\eta_{m}}{2} \\
0 \longrightarrow \text { otherwise }
\end{array},\right.
$$

where $\eta_{0}$ denotes the average value of the distribution and $\eta_{m}$ characterizes the degree of disorder of the environment. However, in the case of RTN the random terms $\vartheta_{k}^{s}(t) \equiv \vartheta_{k}^{R T N}(t)$ behaves as a random bistable fluctuator (RBF) with a constant switching rate $\gamma$. Note that a RBF can be any quantity which switched randomly between two discrete values with a certain switching rate $\gamma$. In this case $\vartheta_{k}^{R T N}(t)$ is characterized by the probability distribution given in Eq. (11). It is worth noting that, depending on the ratio between the switching rate $\gamma$ and the SE coupling constant $g_{k}$, two regimes arise: strong or Markovian RTN and weak or non-Markovian RTN regime. In the case of CN or $1 / f^{\alpha}$ noise, the random parameter $\vartheta_{k}^{s}(t) \equiv \vartheta_{k}^{C N}(t)$ also behaves as a RBF. However, the main difference with the RTN case is that the switching rate of the RBF is not known a priori [16, 17]. In point of fact, here, $\vartheta_{k}^{C N}(t)$ describes a RBF with a switching rate $\gamma$ distributed according to a specific probability law, depending on the colored of the noise. More precisely, the colored of the noise depends upon the value of the exponent $\alpha$ and the cases with $\alpha=1$ and $\alpha=2$ are often called pink and brown noise respectively. The probability distribution of the switching rate $\gamma$ as a function of the parameter $\alpha$ has been found [16, 17] and it reads

$$
\mathrm{P}_{\alpha}(\gamma)=\left\{\begin{array}{l}
\frac{1}{\gamma \ln \left(\gamma_{\max } / \gamma_{\min }\right)} \longrightarrow \alpha=1 \\
\frac{\alpha-1}{\gamma^{\alpha}}\left[\frac{\left(\gamma_{\min } \gamma_{\max }\right)^{\alpha}}{\gamma_{\max }^{\alpha-1}-\gamma_{\min }^{\alpha-1}}\right] \longrightarrow 1<\alpha \leq 1
\end{array}\right.
$$


where $\gamma_{\min }$ and $\gamma_{\max }$ are respectively the minimum and the maximum value of the of the switching rate $\gamma$. Note that, it is also possible to obtain the $1 / f^{\alpha}$ noise by considering a collection of many RBFs [16] but in this work, we will limit ourselves to case of single RBF. As we have already pointed out, since the single qubit Hamiltonian of Eq. (2) is stochastic, the time evolution system is obtained by averaging the time-evolved density $\rho(\{\vartheta\}, t)=\mathcal{U}(\{\vartheta\}, t) \rho(0) \mathcal{U}^{\dagger}(\{\vartheta\}, t)$ both over all the possible realizations of the stochastic $\vartheta_{k}^{s}(t)$ and over all the possible realizations of the switching rate $\gamma$. More precisely the dynamics of the probe system at a given time $t$ can be written as

$$
\rho(t)=\left\langle\langle\rho(\{\vartheta\}, t)\rangle_{\left\{\vartheta_{k}^{s}\right\}}\right\rangle_{\{\gamma\}}=\left\langle\left\langle\mathcal{U}(\{\vartheta\}, t) \rho(0) \mathcal{U}^{\dagger}(\{\vartheta\}, t)\right\rangle_{\left\{\vartheta_{k}^{s}\right\}}\right\rangle_{\{\gamma\}},
$$

where $\rho(0)$ is the input state of the probe system, $\mathcal{U}(\{\vartheta\}, t)=\mathcal{U}_{1}\left(\vartheta_{1}^{s}, t\right) \otimes \mathcal{U}_{2}\left(\vartheta_{2}^{s}, t\right) \otimes \mathcal{U}_{3}\left(\vartheta_{3}^{s}, t\right)$ stands for the global unitary time evolution operator of the probe system for a given noise configuration $\{\vartheta\}=\left\{\vartheta_{1}^{s}, \vartheta_{2}^{s}, \vartheta_{3}^{s}\right\}$. $\langle\ldots\rangle_{\left\{\vartheta_{k}^{s}\right\}}$ and $\langle\ldots\rangle_{\{\gamma\}}$ denote the average over all the possible realizations the stochastic process $\vartheta_{k}^{s}$ and switching rate $\gamma$, respectively. Since the qubits are not interacting, the single qubit time evolution operator $\mathcal{U}_{k}\left(\vartheta_{k}^{s}, t\right)$ can be written (in the unit of $\hbar=1$ ) as

$$
\mathcal{U}_{k}\left(\vartheta_{k}^{s}, t\right)=\exp \left[-\imath \int_{0}^{t} \mathcal{H}_{k}\left(t^{\prime}\right) d t^{\prime}\right]=e^{-\imath \in_{k, 0} t}\left[\begin{array}{cc}
\cos \theta_{k}^{s}(t) & \imath \sin \theta_{k}^{s}(t) \\
\imath \sin \theta_{k}^{s}(t) & \cos \theta_{k}^{s}(t)
\end{array}\right],
$$

where $\theta_{k}^{s}(t)=-g_{k} \int_{0}^{t} \vartheta_{k}^{s}\left(t^{\prime}\right) d t^{\prime}$ stands for the noise phase picked by qubit $k$ during its evolution. In this work, we assumed that the probe system is initially prepared in the GHZ state, that is, $\rho(0)=|G H Z\rangle\langle G H Z|$ with $|G H Z\rangle=\frac{1}{\sqrt{2}}\left(\left|0_{1} 0_{2} 0_{3}\right\rangle+\left|1_{1} 1_{2} 1_{3}\right\rangle\right)$.

As pointed out in the introduction, we quantify the entanglement between the qubits of the probe system by means of the tripartite negativity [35], which is a powerful entanglement estimator arising from the positive partial transpose (PPT) criterion [36] defined as

$$
\mathcal{N}^{(3)}(\rho)=\sqrt[3]{\mathcal{N}_{1-23} \mathcal{N}_{2-13} \mathcal{N}_{3-12}},
$$

where $\mathcal{N}_{\ell-m n}=\sum_{\imath}\left|\lambda_{\imath}\left(\rho^{T_{\ell}}\right)\right|-1$ [with $\lambda_{\imath}\left(\rho^{T_{\ell}}\right)$ the eigenvalues of the partial transpose $\rho^{T_{\ell}}$ of the total density with respect to the qubit $\ell$ ] denotes the bipartite negativity between the subsystem $\ell$ and the compound system $m n$ with $\ell \in\{1,2,3\}$ and $m n \in\{12,13,23\}$. Note that for symmetrical tripartite systems (systems that remain unchanged under the permutation of its parts) the tripartite negativity reduces to the bipartite negativity of any bipartition of the system.

\section{Results and discussions}

In this section, we deal with analytical and numerical results of the evolution of entanglement in all the above mentioned QNC configurations. The entanglement is access by recourse to the tripartite negativity and the probe system is initially prepared in the GHZ state.

\subsection{First $Q N C$ configuration: $(R T N ; S N ; C N)$}

For this configuration, the dynamics of the system as given in Eq. (5) can be written in its more explicit form as

$$
\rho_{c 1}(t)=\int_{\gamma_{\min }}^{\gamma_{\max }} d \gamma_{3} \mathrm{P}_{\alpha}\left(\gamma_{3}\right) \rho\left(\gamma_{3}, t\right)
$$


with

$$
\rho\left(\gamma_{3}, t\right)=\left\langle\left\langle\int_{\tau 1}^{\tau 2} d \theta_{2}^{S N} \mathrm{P}\left(\theta_{2}^{S N}\right) \rho\left(\theta_{1}^{R T N}, \theta_{2}^{S N}, \theta_{3}^{C N}\right)\right\rangle_{\theta_{1}^{R T N}}\right\rangle_{\theta_{3}^{C N}},
$$

141

where $\tau 1=\eta_{0}-\frac{\eta_{m}}{2}, \tau 2=\eta_{0}+\frac{\eta_{m}}{2}$ and

$$
\langle\ldots\rangle_{\left\{\theta_{k}^{s}\right\}}=\int d \theta_{k}^{s}(\ldots) \mathrm{P}\left(\theta_{k}^{s}\right)
$$

In Eq. (10), $\mathrm{P}\left(\theta_{k}^{s}\right), s=\mathrm{RTN}(\mathrm{CN})$ is the probability distribution of the noise phase $\theta_{k}^{s}$ whose explicit form can be written as [37 39$]$

$$
\begin{aligned}
& \mathrm{P}\left(\theta_{k}^{s}, t\right)=\frac{1}{2} e^{-\gamma_{k} t} \\
& \times\left\{\left[\delta\left(\theta_{k}^{s}-g_{k} t\right)+\delta\left(\theta_{k}^{s}+g_{k} t\right)\right]+\frac{\gamma_{k}}{g_{k}}\left[\begin{array}{c}
\Theta\left(\theta_{k}^{s}+g_{k} t\right)+ \\
+\Theta\left(\theta_{k}^{s}-g_{k} t\right)
\end{array}\right]\right\} \\
& \times\left[\frac{I_{1}\left(\theta_{k}^{s} t \sqrt{1-\left(\theta_{k}^{s} / g_{k} t\right)^{2}}\right)}{\sqrt{1-\left(\theta_{k}^{s} / g_{k} t\right)^{2}}}+I_{0}\left(\gamma_{k} t \sqrt{1-\left(\theta_{k}^{s} / g_{k} t\right)^{2}}\right)\right],
\end{aligned}
$$

where $\delta(x)$ is the Dirac delta function, $I_{k}(x)$ is the modified Bessel function and $\Theta(x)$ is the Heaviside step function. Let recall that in the case of RTN the switching $\gamma_{k}$ is constant, that is, $\gamma_{k} \equiv \gamma$ whereas in the case of $\mathrm{CN}, \gamma_{k}$ is no longer constant and is distributed according to the probability law of Eq. (3). Once the calculations are performed, we obtain the following matrix

$$
\begin{aligned}
\rho_{c 1}(t) & =\mathcal{Y}_{c 1}(t)(|000\rangle+|111\rangle)\left(\left\langle000|+\langle 111|)+\mathcal{K}_{c 1}(t)(|001\rangle+|110\rangle) \times\right.\right. \\
& \times\left(\left\langle001|+\langle 110|)+\mathcal{L}_{c 1}(t)(|010\rangle+|101\rangle)(\langle 010|+\langle 101|)+\right.\right. \\
& +\mathcal{F}_{c 1}(t)(|100\rangle+|011\rangle)(\langle 100|+\langle 011|),
\end{aligned}
$$

where

$$
\begin{aligned}
\mathcal{Y}_{c 1}(t) & =\frac{1}{8}(\Phi(t) \Omega(t)+\Phi(t) \Lambda(t)+\Omega(t) \Lambda(t)+1), \\
\mathcal{K}_{c 1}(t) & =-\frac{1}{8}(\Phi(t) \Omega(t)+\Phi(t) \Lambda(t)-\Omega(t) \Lambda(t)+1), \\
\mathcal{L}_{c 1}(t) & =\frac{1}{8}(\Phi(t) \Omega(t)-\Phi(t) \Lambda(t)-\Omega(t) \Lambda(t)+1),
\end{aligned}
$$

and

$$
\mathcal{F}_{c 1}(t)=-\frac{1}{8}(\Phi(t) \Omega(t)-\Phi(t) \Lambda(t)+\Omega(t) \Lambda(t)+1) .
$$

The time dependent functions $\Phi(t), \Omega(t)$ and $\Lambda(t)$ are the decoherence factors induced by the RTN, SN and $\mathrm{CN}$, respectively. They are defined as follows

$$
\Omega(t)=\left\{\begin{array}{c}
e^{-\gamma t}\left[\cosh (\delta t)+\frac{\gamma}{\delta} \sinh (\delta t)\right] \rightarrow \gamma>2 g, \delta=\sqrt{\gamma^{2}-4 g^{2}} \\
e^{-\gamma t}\left[\cos (\delta t)+\frac{\gamma}{\delta} \sin (\delta t)\right] \rightarrow \gamma<2 g . \delta=\sqrt{4 g^{2}-\gamma^{2}}
\end{array} .\right.
$$




$$
\Phi(t)=\frac{\sin \left(g t \eta_{m}\right)}{g t \eta_{m}} \cos \left(2 g t \eta_{0}\right), \text { and } \Lambda(t)=\int_{\gamma_{\min }}^{\gamma_{\max }} d \gamma \mathrm{P}_{\alpha}(\gamma) \Omega(\gamma, t)
$$

For this QNC configuration, the analytical expression of the tripartite negativity obtained from Eq. (7) can be written as follows

$$
\mathcal{N}_{c 1}^{(3)}(t)=\sqrt[3]{\frac{1}{8} \prod_{\mu=1}^{3} \varphi_{\mu}(t) \prod_{(\mu, \nu)}\left(\varphi_{\mu}(t)+\varphi_{\nu}(t)+\left|\varphi_{\mu}(t)-\varphi_{\nu}(t)\right|\right)},
$$

with $(\mu, \nu)=\{(1,2),(1,3),(2,3)\}, \varphi_{1}(t)=\Omega(t), \varphi_{2}(t)=\Lambda(t)$ and $\varphi_{3}(t)=\Phi(t)$. In Fig. 11 we report the evolution of the tripartite negativity as a function of the scaled time $\tau=g t$, in the case of Markov (weak coupling regime) and non-Markov (strong coupling regime) RTN and both for the case of pink $(\alpha=1)$ and brown $(\alpha=2)$ noise. Note that the integral $\Lambda(t)$ in the analytical expression of the tripartite negativity has been computed numerically in the scaled range $\left[\gamma_{\min }, \gamma_{\max }\right] / g=\left[10^{-2}, 10^{2}\right]$. We see that the evolution
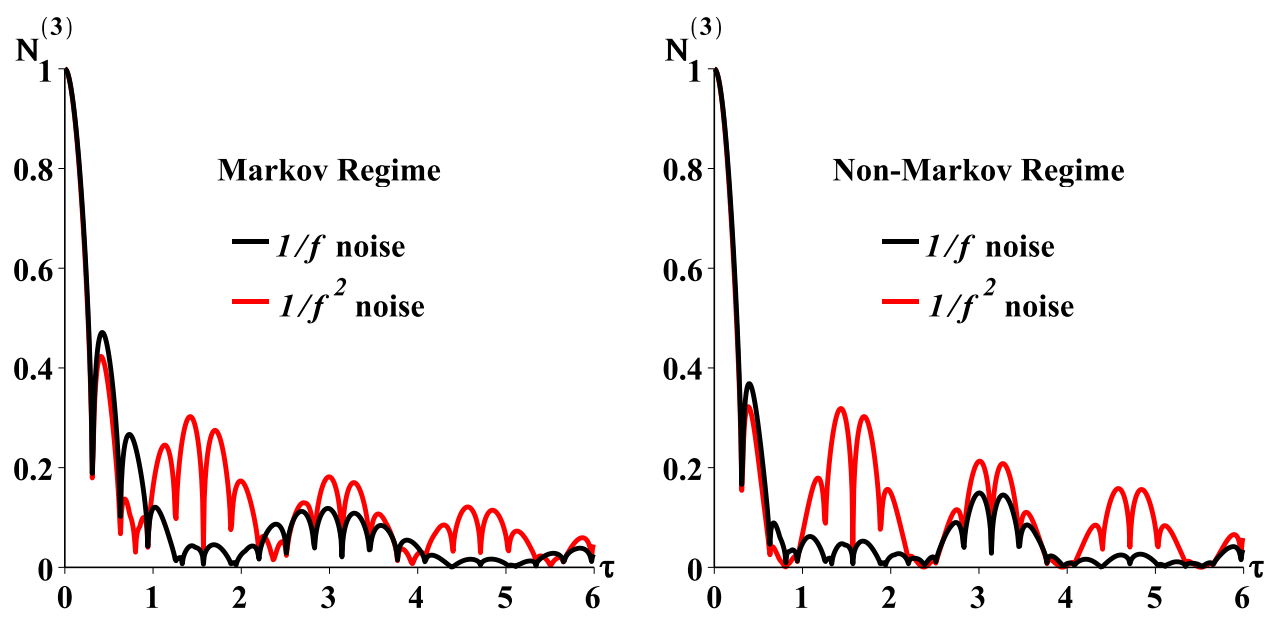

Figure 1: (Color online) Evolution of the tripartite negativity as a function of the scaled time $\tau=g t$ in the case of Markov (left) and Non-Markov (rigth) RTN, both for pink (black solid line curves) and brown (red solid line curves) noise, with the following parameters: $\eta_{m}=10, \eta_{0}=0, \gamma / g=10$ (weak coupling regime), $\gamma / g=0.1$ (strong coupling regime) and $\left[\gamma_{\min }, \gamma_{\max }\right] / g=\left[10^{-2}, 10^{2}\right]$.

of entanglement of the probe system decays with damped oscillations to zero. On the other hand, we see that the evolution of entanglement is strongly affected not only by the colored of the noise (pink or brown) but also by the Markov or non-Markov character of the RTN. In point of fact, we observe from both figures (Markov and non-Markov regime) that for $0 \leq \tau \leq 1$, both pink and brown noises have almost the same destructive influence on the entanglement of the probe system. However, for $\tau>1$ we observe that the pink noise has more destructive influence on the evolution of system with respect to the brown noise. In other words, the pink noise is more fatal to the survival of entanglement than the brown noise, regardless of the Markov or non-Markov character of the RTN. Moreover, we observe both in the case of pink and brown noise that the residual amount of entanglement in the system seems to higher in the Markov regime than in the non-Markov one.

\subsection{Second and third $Q N C$ configuration: $(S N ; S N ; C N),(C N ; S N ; C N)$}

Here, we investigate how entanglement is affected when the RTN is switched either by the SN or CN. This should allow us to evaluate the destructive influence of the presence of the SN as well as CN by 
with

$$
\rho\left(\gamma_{3}, t\right)=\left\langle\int_{\tau 1}^{\tau 2} \int_{\tau 1}^{\tau 2} d \theta_{1}^{S N} d \theta_{2}^{S N} \mathrm{P}\left(\theta_{1}^{S N}\right) \mathrm{P}\left(\theta_{2}^{S N}\right) \rho\left(\theta_{1}^{S N}, \theta_{2}^{S N}, \theta_{3}^{C N}\right)\right\rangle_{\theta_{3}^{C N}}
$$

176 and

$$
\rho_{c 3}(t)=\int_{\gamma_{\min }}^{\gamma_{\max }} \int_{\gamma_{\min }}^{\gamma_{\max }} d \gamma_{1} \mathrm{P}_{\alpha}\left(\gamma_{1}\right) d \gamma_{3} \mathrm{P}_{\alpha}\left(\gamma_{3}\right) \rho\left(\gamma_{1}, \gamma_{3}, t\right)
$$

177 with

$$
\rho\left(\gamma_{1}, \gamma_{3}, t\right)=\left\langle\left\langle\int_{\tau 1}^{\tau 2} d \theta_{2}^{S N} \mathrm{P}\left(\theta_{2}^{S N}\right) \rho\left(\theta_{1}^{R T N}, \theta_{2}^{S N}, \theta_{3}^{C N}\right)\right\rangle_{\theta_{1}^{C N}}\right\rangle_{\theta_{3}^{C N}} .
$$

and

$$
\begin{aligned}
\rho_{c 3}(t) & =\mathcal{Y}_{c 3}(t)(|000\rangle+|111\rangle)\left(\left\langle000|+\langle 111|)+\mathcal{K}_{c 3}(t)(|001\rangle+|110\rangle) \times\right.\right. \\
& \times\left(\left\langle001|+\langle 110|)+\mathcal{L}_{c 3}(t)(|010\rangle+|101\rangle)(\langle 010|+\langle 101|)+\right.\right. \\
& +\mathcal{L}_{c 3}(t)(|100\rangle+|011\rangle)(\langle 100|+\langle 011|),
\end{aligned}
$$

where

$$
\begin{gathered}
\mathcal{Y}_{c 2}(t)=\frac{1}{8}\left(\Phi^{2}(t)+2 \Phi(t) \Lambda(t)+1\right), \mathcal{K}_{c 2}(t)=\frac{1}{8}\left(1-\Phi^{2}(t)\right), \mathcal{L}_{c 2}(t)=\left.\mathcal{Y}_{c 2}(t)\right|_{\Phi=-\Phi} \\
\mathcal{Y}_{c 3}=\frac{1}{8}\left(\Lambda^{2}(t)+2 \Phi(t) \Lambda(t)+1\right), \mathcal{K}_{c 3}(t)=\left.\mathcal{Y}_{c 3}(t)\right|_{\Lambda=-\Lambda}, \mathcal{L}_{c 3}(t)=\frac{1}{8}\left(1-\Lambda^{2}(t)\right) .
\end{gathered}
$$

with $\Phi(t)$ and $\Lambda(t)$ given in Eq. (14). From these density matrices, we can compute from Eq. (7) the tripartite negativities as

$$
\mathcal{N}_{c 2}^{(3)}(t)=\Phi(t) \sqrt[3]{\Lambda(t)\left(\frac{\Phi(t)+\Lambda(t)+|\Phi(t)-\Lambda(t)|}{2}\right)^{2}},
$$


and

$$
\mathcal{N}_{c 3}^{(3)}(t)=\Lambda(t) \sqrt[3]{\Phi(t)\left(\frac{\Phi(t)+\Lambda(t)+|\Phi(t)-\Lambda(t)|}{2}\right)^{2}} .
$$

Note unlike the first configuration case, these expressions depend only on $\Phi(t)$ and $\Lambda(t)$ which are the decoherence factors due to the SN and CN, respectively. It is worth nothing that in the limit of sufficiently long but finite times, both $\Phi(t)$ and $\Lambda(t)$ tend to zero and, as a consequence, the entanglement initially present in the probe system is completely suppressed. In figure 2, we plot the evolution of $\mathcal{N}_{c 2}^{(3)}(t)$ and $\mathcal{N}_{c 3}^{(3)}(t)$ as a function of the scaled time in the case of pink (left) and brown (right) noise. We observed
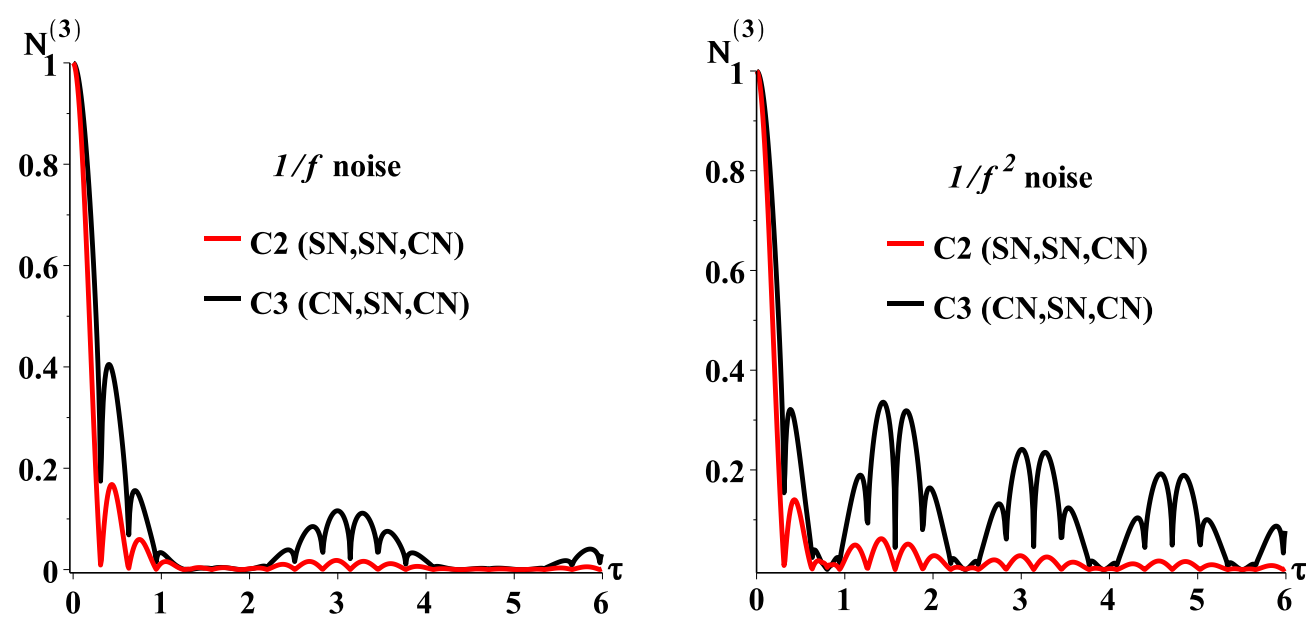

Figure 2: (Color online) Evolution of the tripartite negativity as a function of the scaled time $\tau$ in the case of pink (left) and brown (rigth) noise, both for the second (red solid line curves) and third (black solid line curves) QNC configurations, with the following parameters: $\eta_{m}=10, \eta_{0}=0$ and $\left[\gamma_{\min }, \gamma_{\max }\right] / g=\left[10^{-2}, 10^{2}\right]$.

immediately that when the RTN is switched by the SN (second QNC configuration), the rate of degradation of entanglement is increases with respect to case of the first QNC configuration (Fig. 1), regardless of the Markov or non-Markov regime. This clearly indicate that the SN has more destructive effects on the probe system than the RTN. Another information that one can observe in this figure is that the CN, independently on the colore considered, is less detrimental than the SN. Indeed, we observe from Fig 1 that whatever the colore of the $\mathrm{CN}$ is, the curves obtained for the second QNC configuration are either slightly or completely below those of the third QNC configuration. Overall, Fig. 2 clearly shows that the SN is more fatal to the probe system than the RTN and CN. However, for RTN and CN no conclusion can be draw yet.

\subsection{Fourth and fifth $Q N C$ configuration: $(R T N ; R T N ; C N),(R T N ; C N ; C N)$}

Here, we analyze the situation in which the coupling of the second qubit of the probe system is switched from SN to either RTN (fourth QNC configuration) or CN (fifth QNC configuration). Both configurations shall enable us to illustrate how the RTN and CN affect the probe system. For both configurations, the evolution of the system is described by the following density matrices

$$
\rho_{c 4}(t)=\int_{\gamma_{\min }}^{\gamma_{\max }} d \gamma_{3} \mathrm{P}_{\alpha}\left(\gamma_{3}\right) \rho\left(\gamma_{3}, t\right)
$$

with

$$
\rho\left(\gamma_{3}, t\right)=\left\langle\left\langle\left\langle\rho\left(\theta_{1}^{R T N}, \theta_{2}^{R T N}, \theta_{3}^{C N}\right)\right\rangle_{\theta_{1}^{R T N}}\right\rangle_{\theta_{2}^{R T N}}\right\rangle_{\theta_{3}^{C N}},
$$


and

with

$$
\rho_{c 5}(t)=\int_{\gamma_{\min }}^{\gamma_{\max }} \int_{\gamma_{\min }}^{\gamma_{\max }} d \gamma_{2} \mathrm{P}_{\alpha}\left(\gamma_{2}\right) d \gamma_{3} \mathrm{P}_{\alpha}\left(\gamma_{3}\right) \rho\left(\gamma_{1}, \gamma_{3}, t\right)
$$

$$
\rho\left(\gamma_{2}, \gamma_{3}, t\right)=\left\langle\left\langle\left\langle\rho\left(\theta_{1}^{R T N}, \theta_{2}^{C N}, \theta_{3}^{C N}\right)\right\rangle_{\theta_{1}^{R T N}}\right\rangle_{\theta_{2}^{C N}}\right\rangle_{\theta_{3}^{C N}} .
$$

After performing calculations, we find that the density matrix $\rho_{c 4}(t)$ has the same functional expression of $\rho_{c 2}(t)$ (Eq. (20) ), but with the following coefficients

$$
\begin{aligned}
& \mathcal{Y}_{c 2}(t) \rightarrow \mathcal{Y}_{c 4}(t)=\frac{1}{8}\left(\Omega^{2}(t)+2 \Omega(t) \Lambda(t)+1\right), \mathcal{K}_{c 2}(t) \rightarrow \mathcal{K}_{c 4}(t)=\frac{1}{8}\left(1-\Omega^{2}(t)\right) \\
& \mathcal{F}_{c 2}(t) \rightarrow \mathcal{F}_{c 4}(t)=\left.\mathcal{Y}_{c 4}(t)\right|_{\Omega=-\Omega}
\end{aligned}
$$

However, the time-evolved density matrix $\rho_{c 5}(t)$ can be written in the three-qubit computational basis as

$$
\begin{aligned}
\rho_{c 5}(t) & =\mathcal{Y}_{c 5}(t)(|000\rangle+|111\rangle)\left(\left\langle000|+\langle 111|)+\mathcal{K}_{c 5}(t)(|001\rangle+|110\rangle) \times\right.\right. \\
& \times\left(\left\langle001|+\langle 110|)+\mathcal{K}_{c 5}(t)(|010\rangle+|101\rangle)(\langle 010|+\langle 101|)+\right.\right. \\
& +\mathcal{F}_{c 5}(t)(|100\rangle+|011\rangle)(\langle 100|+\langle 011|),
\end{aligned}
$$

with

$$
\begin{aligned}
& \mathcal{Y}_{c 5}(t)=\frac{1}{8}\left(\Lambda^{2}(t)+2 \Omega(t) \Lambda(t)+1\right), \mathcal{K}_{c 5}(t)=\frac{1}{8}\left(1-\Lambda^{2}(t)\right), \\
& \mathcal{F}_{c 5}(t)=\left.\mathcal{Y}_{c 5}(t)\right|_{\Lambda=-\Lambda}
\end{aligned}
$$

where $\Omega(t)$ and $\Lambda(t)$ are given in Eq. (13) and Eq. (14) respectively. From the definition of the tripartite negativity expressed in Eq. (7), we obtain

$$
\mathcal{N}_{c 4}^{(3)}(t)=\lim _{\Phi \rightarrow \Omega} \mathcal{N}_{c 2}^{(3)}(t)=\Omega(t) \sqrt[3]{\Lambda(t)\left(\frac{\Omega(t)+\Lambda(t)+|\Omega(t)-\Lambda(t)|}{2}\right)^{2}},
$$

and

$$
\mathcal{N}_{c 5}^{(3)}(t)=\Lambda(t) \sqrt[3]{\Omega(t)\left(\frac{\Lambda(t)+\Omega(t)+|\Omega(t)-\Lambda(t)|}{2}\right)^{2}} .
$$

In figure 3 we report the evolution of the tripartite negativities as a function of the dimensionless time $\tau$, for the cases of pink and brown noise and both for Markov and non-Markov RTN. The observation of this figure clearly confirms what we have already pointed out that the SN is more fatal to the probe system than the CN and RTN, regardless of the Markov or non-Markov character of the RTN as well as the color of the CN. Indeed, by comparing Fig. 3 with Figs. 1 and 2, we observe that when the second qubit of the probe system is no longer affected by the SN but rather by the RTN or CN, the entanglement is less degraded. This figure also reveals that the pink noise is more fatal to the probe system than both Markov and non-Markov RTN. However, the situation is totally reversed in the case of brown noise. In other words, the brown noise is less fatal to system than the RTN, regardless of the Markov or non-Markov character of the RTN. In point of fact, as it can clearly be seen in Fig. 3 the curves obtained both in the weak and strong coupling regime (Markov and non-Markov RTN) in the fourth QNC configuration are either slightly or completely above (pink noise)/below (brown noise) those obtained in the fifth QNC configuration. So, at this level we can say that we have achieved the main objective of this work, which was to classify the SN, CN and RTN with respect to their destructive influence on a quantum system. Nevertheless, in the following we will investigate two other QNC configurations to reinforce what we have already concluded in both previous subsections. 

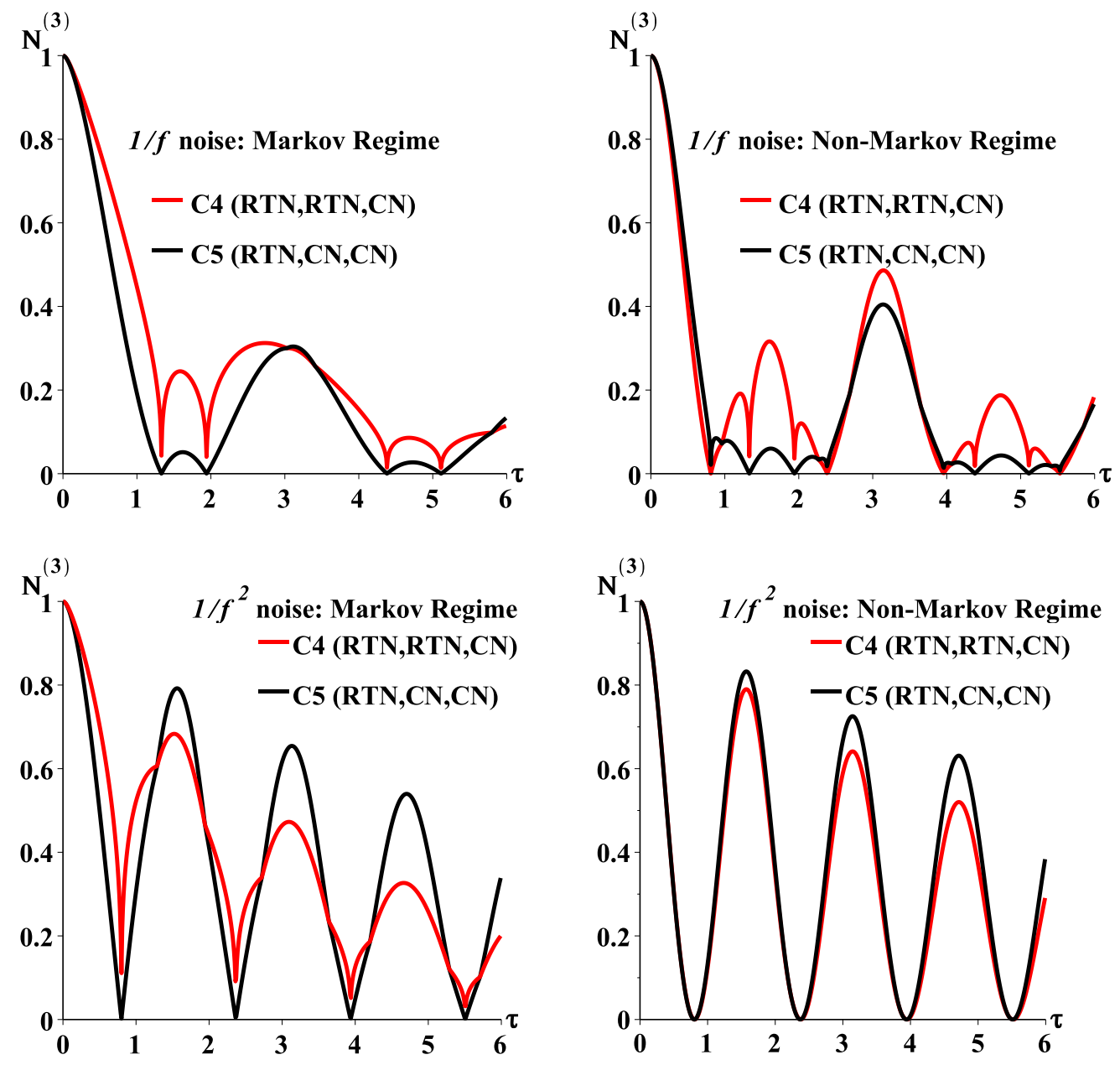

Figure 3: (Color online) Upper panels: evolution of the tripartite negativity as a function of the scaled time $\tau$ in the case of: (left) pink noise and Markov RTN; (right) pink noise and Non-Markov RTN, both for the fourth (black solid line curves) and fifth (red solid line curves) QNC configuration, with the following parameters: $\gamma / g=10$ (weak coupling regime), $\gamma / g=0.1$ (strong coupling regime) and $\left[\gamma_{\min }, \gamma_{\max }\right] / g=\left[10^{-2}, 10^{2}\right]$. Lower panels: Same as in the upper panels but in the case of brown noise.

\subsection{Sixth and seventh QNC configuration: (RTN;SN;RTN), (RTN;SN;SN)}

As we have already mentioned above, if we limit ourselves to previous analyzed QNC we can already classify the SN, CN and RTN with respect to their detrimental effects. However, in order to reinforce what has already been concluded, here we are going to investigate the situation in which the coupling of the third qubit of the probe system is switched from CN to either RTN or SN. In such a situation, the dynamics of the probe system at a given time $t$ can be written as

$$
\rho_{c 6}(t)=\left\langle\left\langle\int_{\tau 1}^{\tau 2} d \theta_{2}^{S N} \mathrm{P}\left(\theta_{2}^{S N}\right) \rho\left(\theta_{1}^{R T N}, \theta_{2}^{S N}, \theta_{3}^{R T N}\right)\right\rangle_{\theta_{1}^{R T N}}\right\rangle_{\theta_{3}^{R T N}},
$$

and

$$
\rho_{c 7}(t)=\left\langle\int_{\tau 1}^{\tau 2} \int_{\tau 1}^{\tau 2} d \theta_{2}^{S N} d \theta_{3}^{S N} \mathrm{P}\left(\theta_{2}^{S N}\right) \mathrm{P}\left(\theta_{3}^{S N}\right) \rho\left(\theta_{1}^{R T N}, \theta_{2}^{S N}, \theta_{3}^{S N}\right)\right\rangle_{\theta_{1}^{R T N}} .
$$


Once performing the calculations, we find again that the density matrices $\rho_{c 6}(t)$ and $\rho_{c 7}(t)$ have the same functional expressions of $\rho_{c 3}(t)$ (Eq. (21)) and $\rho_{c 5}(t)$ (Eq. (28)), respectively, but with the following coefficients

$$
\begin{aligned}
& \mathcal{Y}_{c 3}(t) \rightarrow \mathcal{Y}_{c 6}(t)=\frac{1}{8}\left(\Omega^{2}(t)+2 \Omega(t) \Phi(t)+1\right), \mathcal{K}_{c 3}(t) \rightarrow \mathcal{K}_{c 6}(t)=\left.\mathcal{Y}_{c 6}(t)\right|_{\Omega=-\Omega}, \\
& \mathcal{Y}_{c 5}(t) \rightarrow \mathcal{Y}_{c 7}(t)=\frac{1}{8}\left(\Phi^{2}(t)+2 \Omega(t) \Phi(t)+1\right), \mathcal{F}_{c 5}(t) \rightarrow \mathcal{F}_{c 7}(t)=\left.\mathcal{Y}_{c 7}(t)\right|_{\Phi=-\Phi}, \\
& \mathcal{L}_{c 3}(t) \rightarrow \mathcal{L}_{c 6}(t)=\frac{1}{8}\left(1-\Omega^{2}\right), \mathcal{K}_{c 5}(t) \rightarrow \mathcal{K}_{c 7}(t)=\frac{1}{8}\left(1-\Phi^{2}\right) .
\end{aligned}
$$

From the definition of the tripartite negativity expressed in Eq. (7), we obtain

$$
\mathcal{N}_{c 6}^{(3)}(t)=\lim _{\Lambda \rightarrow \Omega} \mathcal{N}_{c 3}^{(3)}(t)=\Omega(t) \sqrt[3]{\Phi(t)\left(\frac{\Omega(t)+\Phi(t)+|\Omega(t)-\Phi(t)|}{2}\right)^{2}},
$$

and

$$
\mathcal{N}_{c 7}^{(3)}(t)=\lim _{\Lambda \rightarrow \Phi} \mathcal{N}_{c 5}^{(3)}(t)=\Phi(t) \sqrt[3]{\Omega(t)\left(\frac{\Omega(t)+\Phi(t)+|\Omega(t)-\Omega(t)|}{2}\right)^{2}} .
$$

In figure 4, we report the evolution of $\mathcal{N}_{c 6}^{(3)}(t)$ and $\mathcal{N}_{c 7}^{(3)}(t)$ as a function of the scaled time $\tau$ in the case of Markov (left) and non-Markov (right) RTN. We observe both in the Markov and non-Markov regime that

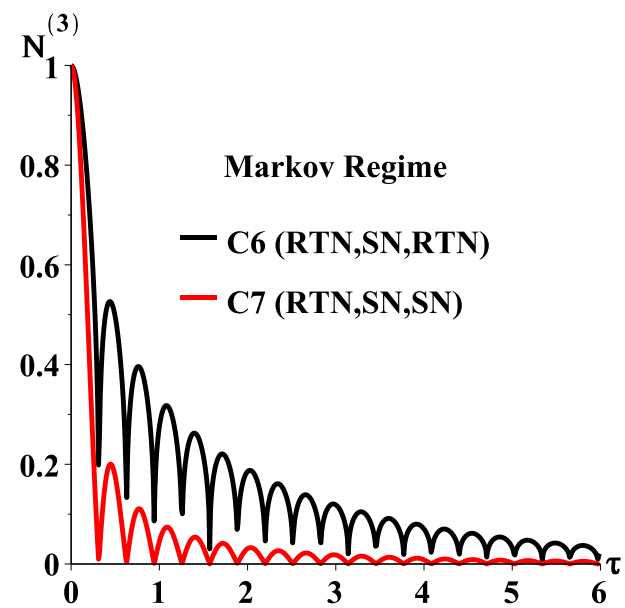

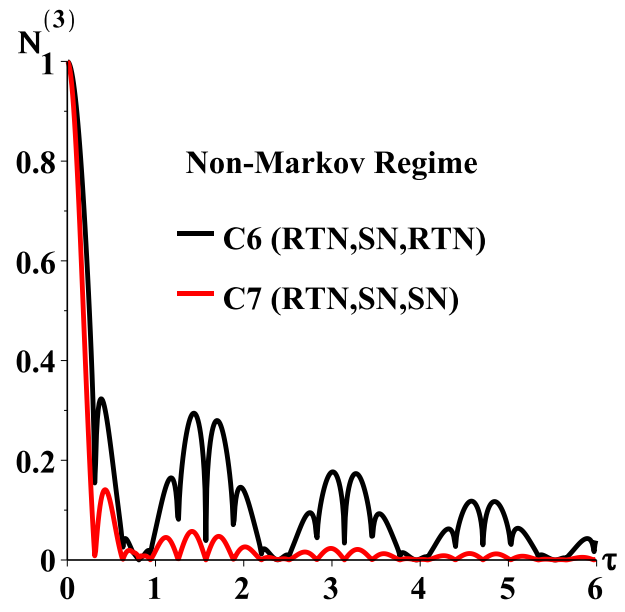

Figure 4: (Color online) Evolution of the tripartite negativity as a function of the scaled time in the case of Markov (left) and non-Markov (rigth) RTN, both for the sixth (black solid line curves) and fifth (red solid line curves) QNC configurations, with the following parameters: $\eta_{m}=10, \eta_{0}=0, \gamma / g=10$ (weak coupling regime), and $\gamma / g=0.1$ (strong coupling regime).

the probe system is less influence by the overall external noise when its third qubit is coupled to the RTN than when it is coupled to the SN, demonstrating that the SN is more harmful than the RTN. This result clearly reinforces what has been previously found. On the other hand, by comparing the left panel of Fig. 2 with Fig. (4, we clearly observe that the pink noise is more detrimental than the Markov and non-Markov RTN. Moreover, by comparing the left panel of Fig. 2 with its right panel, we observe (on the whole) that entanglement is less degraded by the Markov RTN with respect to the non-Markov RTN. 


\section{Conclusions}

In this paper we have investigate the classification of three different classical non-Gaussian noises, namely the static, colored and random telegraph noise, with respect to their negative effects on the evolution of entanglement, using a system of three noninteracting qubits as probe. The probe system has been initially prepared in the GHZ state and seven QNC configurations have been analyzed in detail. Moreover, the probe system has been described by a stochastic Hamiltonian and the dynamics of the system for each QNC configuration has been computed by performing an ensemble average over all the possible realizations of the stochastic processes. Using the tripartite negativity as measure of entanglement, the evolutions of entanglement in these configurations have been compared to each other to evaluate the detrimental effects of the presence/absence of each of kind of noise considered.

In all QNC configurations analyzed, we found that the evolution of entanglement is severely destroyed by the external noise. On the other hand, we found that depending on the QNC configuration as well the noise parameters (Markovian or non-Markovian regime/pink or brown noise) considered, the entanglement decay rate is slowed down or quickened. By comparing the evolutions of entanglement obtained in the first, second and third QNC configuration to each other, we have found that the SN is more fatal to the survival of entanglement than the RTN and CN. In the same way, the evolutions of entanglement in the first, fourth and fifth QNC configuration have been compared to each other and we have found that depending on the noise color considered, the RTN may be more fatal to the probe system than the CN. In point of fact, we found that the RTN is less detrimental to the survival of entanglement of the probe system than the pink noise and that the situation is totally reversed in the case of brown noise. Finally, the evolutions of entanglement in the sixth and seventh QNC configuration have been compared with those in the first QNC configuration and once again we have found that whatever the regime (Markov or non-Markov) considered is, SN is more fatal than the RTN.

Overall, we found that these noises can be classified in descending order (according to their detrimental influence) as: SN $>$ pink noise $>$ RTN (both Markovian and non-Markovian) $>$ brown noise (where ">"means more detrimental than). Hence, we believe that our investigation can be useful for the engineering of classical non-Gaussian distributed environmental noise.

\section{Acknowledgement}

This research did not receive any specific grant from funding agencies in the public, commercial, or not-for-profit sectors.

\section{References}

[1] Schroedinger, Die Naturwissenschaften 23 (1935) 807-8012.

[2] R. Horodecki, P. Horodecki, M. Horodecki, K. Horodecki, Rev. Mod. Phys. 81 (2009) 865.

[3] A. K. Ekert, Phys. Rev. Lett. 67 (1991) 661.

[4] N. Gisin, et al., Rev. Mod. Phys. 74 (2002) 145.

[5] M. Nielsen, I. Chuang, Quantum computation and quantum information, Cambridge University Press, England (2000).

[6] C. H. Bennett, Phys. Rev. Lett. 68 (1992) 3121.

[7] C. H. Bennett, G. Brassard, C. Crepeau, R. Jozsa, A. Peres, W. Wootters, Phys. Rev. Lett. 70 (1993) 1895.

[8] R. Vasile, S. Olivares, M. G. A. Paris, S. Maniscalco, Phys. Rev. A 80 (2009) 062324.

[9] J. P. Paz, A. J. Roncaglia, Phys. Rev. Lett. 100 (2008) 220401.

[10] J. Helm, W. T. Strunz, Phys. Rev. A 80 (2009) 042108.

[11] J. Helm, W. T. Strunz, S. Rietzler, L. E. Wuringer, Phys. Rev. A 83 (2011) 042103.

[12] W. M. Witzel, K. Young, S. D. Sarma, Phys. Rev. B 90 (2014) 115431.

[13] W. T. Strunz, L. Diosi, N. Gisin, Phys. Rev. Lett. 82 (1999) 1801.

[14] J. T. Stockburger, H. Grabert, Phys. Rev. Lett. 88 (2002) 170407.

[15] D. Crow, R. Joynt, Phys. Rev. A 89 (2014) 042123.

[16] C. Benedetti, F. Buscemi, P. Bordone, M. G. A. Paris, Phys. Rev. A 87 (2013) 052328.

[17] C. Benedetti, M. G. A. Paris, F. Buscemi, P. Bordone, Time-evolution of entanglement and quantum discord of bipartite systems subject to $1 / f^{\alpha}$ noise., Proceedings of the 22 nd International Conference on Noise and Fluctuations (ICNF) 323 (2013) 6578952. doi:10.1109/ICNF

[18] C. Benedetti, F. Buscemi, P. Bordone, M. G. A. Paris, Int. J. Quant. Inf. 10 (2012) 1241005. 
[19] M. A. C. Rossi, C. Benedetti, M. G. A. Paris, Int. J. Quantum Inf. 12 (2014) 1560003.

[20] F. Buscemi, P. Bordone, Phys. Rev. A 87 (2013) 042310.

295 [21] L. T. Kenfack, M. Tchoffo, M. N. Jipdi, J. C. Fuoukeng, L. C. Fai, arXiv:1707.02762.

296 [22] L. T. Kenfack, M. Tchoffo, G. C. Fouokeng, L. C. Fai, Int. J. Quant. Inf. 15 (2017) 1750038.

297 [23] L. T. Kenfack, Tchoffo, L. C. Fai, Eur. Phys. J. Plus. 132 (2017) 91.

298 [24] L. T. Kenfack, Tchoffo, L. C. Fai, G. C. Fouokeng, Physica B 511 (2017) 123.

299 [25] M. Tchoffo, L. T. Kenfack, G. C. Fouokeng, L. C. Fai, Eur. Phys. J. Plus. 131 (2016) 380.

[26] T. K. Lionel, T. Martin, F. G. Collince, L. C. Fai, Int. J. Mod. Phys. B 30 (2016) 1750046.

[27] D. Zhou, A. Lang, R. Joynt, Quantum Inf. Process. 9 (2010) 727.

[28] A. De, A. Lang, D. Zhou, R. Joynt, Phys. Rev. A 83 (2011) 042331.

[29] B. Leggio, R. L. Franco, D. O. Soares-Pinto, P. Horodecki, G. Compagno, Phys. Rev. A 92 (2015) 032311.

[30] A. D'Arrigo, G. Benenti, R. L. Franco, G. Falci, . E. Paladino, Int. J. Quant. Inf. 12 (2014) 1461005.

[31] J.-S. Xu, K. Sun, C.-F. Li, et al., Nat. Commun. 4 (2013) 2851.

[32] A. Orieux, A. D'Arrigo, G. Ferranti, R. L. Franco, et al., Sci. Rep. 5 (2015) 8575.

[33] C. Thompson, G. Vemuri, G. S. Agarwal, Phys. Rev. A 82 (2010) 053805.

[34] P. Bordone, F. Buscemi, C. Benedetti, Fluct. Noise Lett. 11 (2012) 1242003.

[35] C. Sabin, G. Garcia-Alcaine, Eur. Phys. J. D 48 (2008) 435.

[36] G. Vidal, R. F. Werner, Phys. Rev. A 65 (2002) 032314.

310

$38]$ E. Paladino, L. Faoro, G. Falci, R. Fazio, Phys. Rev. Lett. 88 (2002) 228304.

[39] G. Falci, A. D'Arrigo, A. Mastellone, E. Paladino, ibid. 94 (2005) 167002. 